\title{
3q27.1 microdeletion causes prenatal and postnatal growth restriction and neurodevelopmental abnormalities
}

\author{
Subit Barua ${ }^{1}$, Elaine M. Pereira ${ }^{2}$, Vaidehi Jobanputra ${ }^{3}$, Kwame Anyane-Yeboa ${ }^{2}$, Brynn Levy ${ }^{3}$ and Jun Liao ${ }^{3^{*}}$ (i)
}

\begin{abstract}
Background: Overlapping microdeletions of chromosome 3q26-3q28 have been reported in eight individuals. The common phenotype observed in these individuals include intrauterine growth restriction, short stature, microcephaly, feeding difficulties, facial dysmorphisms, limb abnormalities and developmental delay. The most striking clinical features shared among all reported cases is prenatal and postnatal growth restriction and neurodevelopmental abnormalities.
\end{abstract}

Case presentation: We identified two additional individuals with overlapping deletions and shared clinical features by high-resolution SNP oligonucleotide microarray, and refined the smallest region of overlap (SRO) to a $1.2 \mathrm{Mb}$ genomic location in chromosome 3q27.1 by reviewing and comparing all published cases. We evaluated the SRO using ACMG/ClinGen current recommendations for classifying copy number variants (CNVs), and discussed the contribution of the genes deleted in the SRO to the abnormal phenotype observed in these individuals.

Conclusions: This study provides further evidence supporting the existence of a novel 3q27.1 microdeletion syndrome and suggests that haploinsufficiency of potential candidate genes, DVL3, AP2M1, and PARL in the SRO in 3q27.1 is responsible for the phenotype.

Keywords: 3q27.1 microdeletion, DVL3, AP2M1, PARL, CNV interpretation

\section{Background}

Intrauterine growth restriction (IUGR) is a condition where fetal growth did not achieve the normal growth expected for the gestational age. It is a leading cause of perinatal mortality and morbidity that needs long-term follow up due to an increase risk for future development of chronic diseases. IUGR can be caused by genetic, epigenetic, metabolic, endocrine, or environmental factors [1-4]. Symmetric IUGR, where weight, length, and head circumference are equally affected, can be secondary to infections, chromosomal variants, or the suboptimal

*Correspondence: j15098@cumc.columbia.edu

${ }^{3}$ Department of Pathology and Cell Biology, Columbia University Medical Center, New York, NY, USA

Full list of author information is available at the end of the article nutritional supply that affect the pregnancy at early stages. Asymmetric IUGR, where the weight is most affected and the head circumference is spared, occurs more commonly at later stages of pregnancy and is commonly from lack of nutrition. When IUGR is identified, the expectant mother is worked up for infectious through TORCH titers and are normally offered invasive testing with a microarray and chromosome analysis to rule out common causes of IUGR. Specifically, there is a strong association of IUGR with chromosomal aberrations. In some micro-duplication/deletion syndromes, IUGR is a major and only manifestation $[5,6]$.

One of the chromosome regions associated with IUGR is located in chromosome 3q26-3q28. Patients with microdeletions in this region are rare and not well described. To date eight cases have been reported in original author(s) and the source, provide a link to the Creative Commons licence, and indicate if changes were made. The images or other third party material in this article are included in the article's Creative Commons licence, unless indicated otherwise in a credit line to the material. If material is not included in the article's Creative Commons licence and your intended use is not permitted by statutory regulation or exceeds the permitted use, you will need to obtain permission directly from the copyright holder. To view a copy of this licence, visit http://creativecommons.org/licenses/by/4.0/. The Creative Commons Public Domain Dedication waiver (http://creativeco mmons.org/publicdomain/zero/1.0/) applies to the data made available in this article, unless otherwise stated in a credit line to the data. 
the literature with deletions varying in size from $\sim 2.0$ to 8.4 Mb [7-12]. Although their breakpoints are not recurrent, these patients share an apparently distinct phenotype including IUGR, microcephaly, short stature, facial abnormities, and feeding difficulties. However, the clinical significance and genetic mechanism of the 3q26q28 microdeletion are not fully established. Here we report two unrelated individuals harboring overlapped microdeletions in this region and sharing clinical features with those reported in the literature. Using high-resolution single nucleotide polymorphism (SNP) microarrays, we narrowed the Smallest Region of Overlap (SRO) to a size of 1.2 Mb at chromosomal band 3q27.1. This SRO region contains 46 genes, 24 of which are OMIM-annotated and eight of which are associated with disease (KLHL24, EIF2B5, DVL3, AP2M1, ALG3, EIF4G1, CLCN2, and THPO).

Genome-wide assessment of copy-number variants (CNVs) is widely applied to assess the clinical significance of pre- and post-natal congenital abnormalities. However, in-between clinical laboratories, the assessment of $\mathrm{CNV}$ classification remains inconsistent due to lack of uniform scoring metrics. To assist clinical laboratories in the accurate and consistent classification of reporting CNVs, ACMG and ClinGen recently published technical standards for CNV interpretation [13]. We applied their recommended quantitative and evidence-based scoring framework to evaluate the deduced 1.2 Mb SRO at chromosomal band 3q27.1 and classified it as a pathogenic deletion.

\section{Case presentation Proband-1}

A G1P0 woman with naturally conceived male fetus was referred for prenatal diagnosis due to second trimester abnormal ultrasound findings (Table 1): $<5 \%$ abdominal circumference, absent nasal bone, placentomegaly and severe oligohydramnios. Fetal measurement revealed a fetal weight at less than the tenth centile, which was consistent with a diagnosis of IUGR. Fetal echocardiogram was concerning for cardiomegaly and hypoplastic aortic arch. The proband was born at 37 weeks and 3 days of gestation via normal spontaneous vaginal delivery with a birth weight of $1785 \mathrm{~g}$. Postnatal echocardiogram ruled out any cardiac anomaly and newborn screening was normal. By 3 months old, the proband continued to have growth parameters below the 5th centile; weight:3.7 kg ( $<3 \mathrm{rd}$ centile); length: $54.5 \mathrm{~cm}$ ( $<3 \mathrm{rd}$ centile); occipitofrontal circumference (OFC) $38.5 \mathrm{~cm}$ (5th centile). He was dysmorphic with microcephaly, mild frontal bossing, bilateral epicanthal folds, hypotelorism, posteriorly rotated ears, flat nasal bridge, micrognathia, high arched palate, left palmer simian crease, and increased muscle tone. By 18 months old he was receiving speech and feeding therapies. Gross motor skills were delayed as he was only beginning to cruise. By 30 months old, he started to walk, and by 3 years old be had 20-30 words. By 3 years of age, the proband started to walk. A neuropsychological evaluation at 4 yo demonstrated the proband was too cognitively limited to complete the exam. The proband's family was not interested in a gastrostomy tube, and he continued to grow at $<3$ rd percentile though he was not a picky eater. By 6 years old, the proband has not been potty trained. At the last evaluation at 8 years old, he continues to be in special education class with services and continues to have trouble with comprehension. His physical exam still demonstrated $<2$ nd percentile for height, weight, and head circumference. Additional findings of dolichocephaly and arachnodactyly were also noted.

\section{Proband-2}

The proband is a female child of non-consanguineous Dominican desecnt. Prenatal ultrasounds at 24 weeks gestational age was concerning for symmetric IUGR where the head circumference was 3 standard deviations below the mean. An infectious and cardiac workup were normal. At 37 weeks gestational age oligohydramnios was noted. The proband was born full term as small for gestational age with weight, length and head circumference all $<2$ nd percentile (weight $1.725 \mathrm{~kg}$, length $44 \mathrm{~cm}$, head circumference $30 \mathrm{~cm}$ ). A post-natal TORCH panel was sent along with CMV urine analysis which were normal. She passed her newborn screen. Though the proband was discharged within $48 \mathrm{~h}$, she was readmitted for hyperbilirubinemia that required phototherapy. At 6 months of age, her growth parameters were only at the 1st percentile. She continued to have failure to gain weight, prompting an admission for further workup when she was 11 months old. A nasogastric tube was placed to help with her feedings. During the admission she had a genetics evaluation. Her exam was notable for microcephaly, epicanthal folds, hypertelorism, an arched palate, and retrognathia. After her hospital discharge, the proband's family had difficulty with follow up involving therapies and sub-specialty providers. She qualified for speech therapy but was not able to attend many of the sessions. Although she qualified for physical therapy, the family declined services once she began to walk at 20 months of age. At 3 years old she has many words but is working on sentences. She remains consistently at less than the 3rd percentile for height, weight, and head circumference. At this time, the patient is constantly encouraged to follow up with services through the school system, and to schedule appointments with appropriate subspecialty providers to help with feeding issues. 


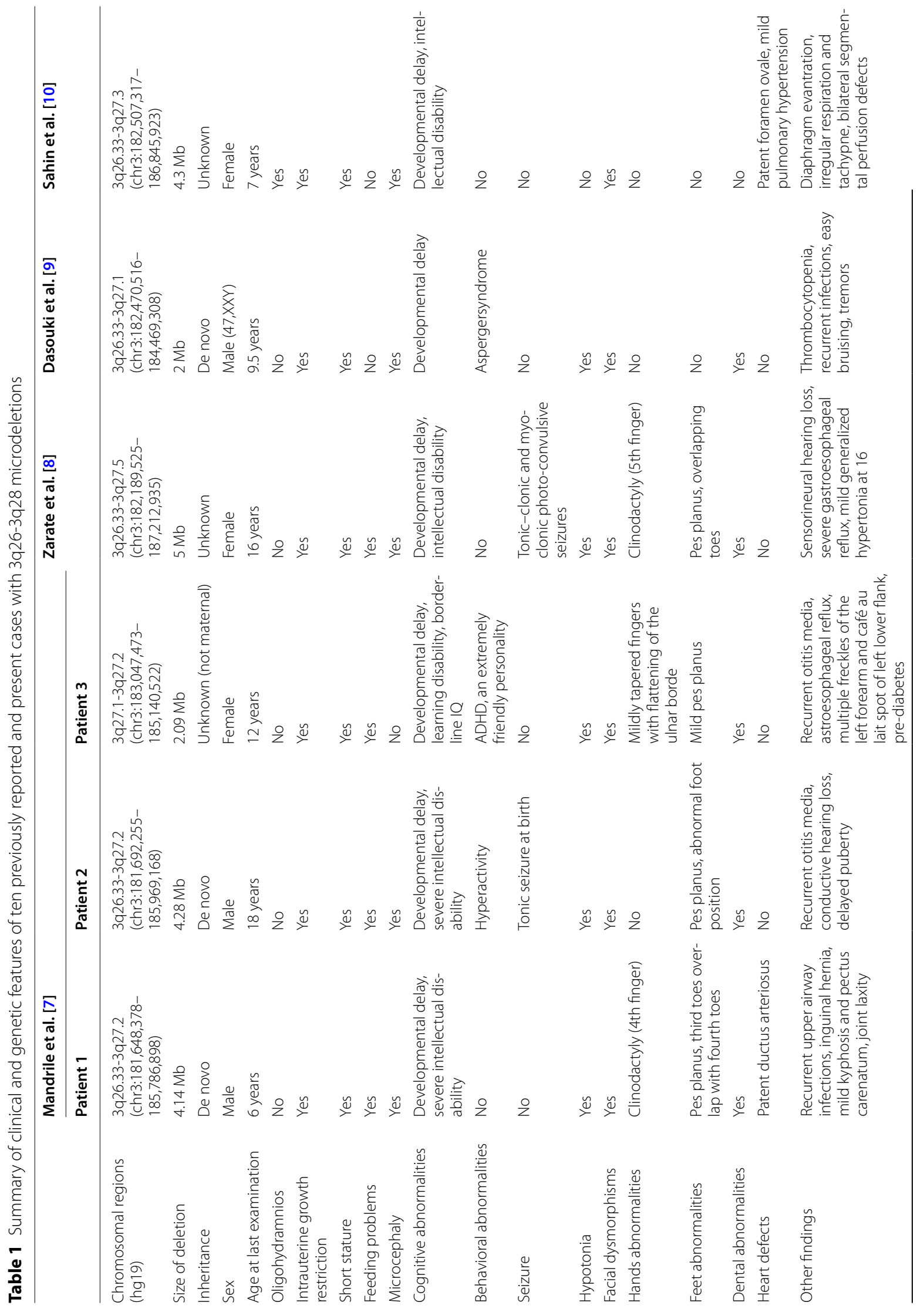




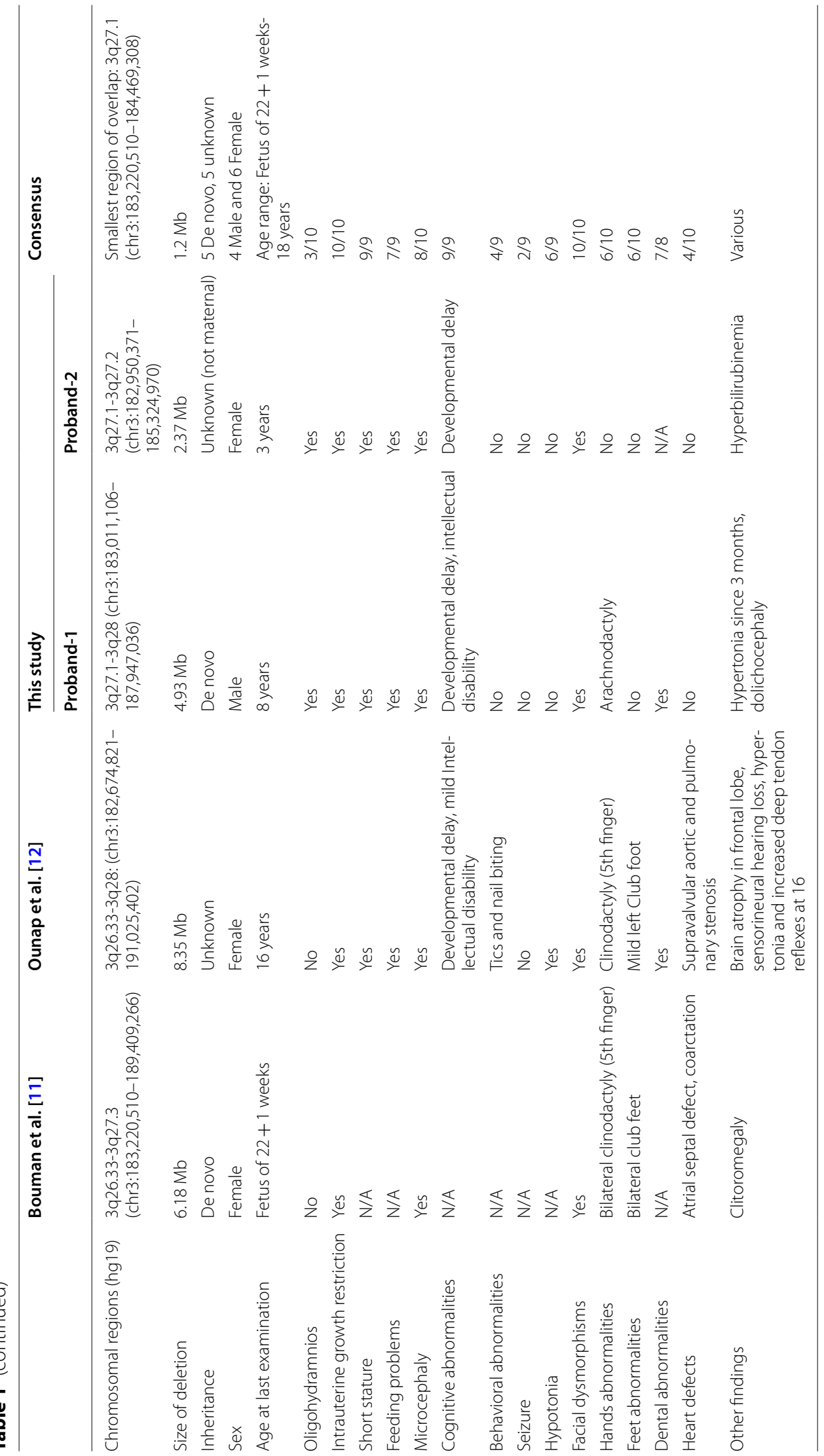




\section{Results}

\section{Proband-1}

SNP Oligonucleotide Microarray Analysis (SOMA) using Affymetrix GeneChip Human Mapping 6.0 SNP array and Affymetrix Chromosome Analysis Suite 3.3 (Affymetrix, Santa Clara, CA) revealed that Proband-1 harbors a $4.93 \mathrm{Mb}$ deletion in genomic coordinates 183,011,106-187,947,036 (hg19) corresponding to chromosomal bands 3q27.1q28 (arr[hg19] 3q27 .1q28(183,011,106_187,947,036)x1; Additional file 1: Fig. $\mathrm{S} 1 \mathrm{a})$. This deleted region contains 122 genes, 53 of which are OMIM-annotated and 20 of which are associated with disease. Maternal SOMA showed that the mother does not have the same deletion in the long arm of chromosome 3 (Additional file 1: Fig. S1b). FISH using a BAC probe RP11-919L13 further confirmed that this deletion is present in the proband but not in the father (Additional file 1: Fig. S1c and d) and is therefore de novo in origin. No other CNVs except for those commonly seen in normal populations were detected in this individual.

\section{Proband-2}

A karyotype analysis of Proband-2 with a resolution level of 525 bands revealed a normal female chromosome complement $(46, \mathrm{XX})$. SOMA in Proband-2 identified a $2.37 \mathrm{Mb}$ deletion (Additional file 1: Fig. S2a) in the chromosomal region 3q27.1q27.2, corresponding to genomic coordinates 182,950,371-185,324,970 (arr[hg19] 3q27 $\left..1 q 27.2\left(182,950,371 \_185,324,970\right) x 1\right)$. This deleted region contains 66 genes, 30 of which are OMIM-annotated and 10 of which are associated with disease. FISH using a BAC probe RP11-919L13 confirmed the presence of this deletion in Proband-2 and excluded its maternal inheritance (Additional file 1: Fig. S2b and c). Father is unavailable for testing. No other CNVs except for those commonly seen in normal populations were detected in this individual.

\section{Discussion and conclusions}

In the literature, there are eight previously reported cases carrying 3q26-3q28 microdeletions with sizes of 2-8.4 Mb that overlapped with the deleted chromosomal regions in two patients from this study [7-12]. The clinical phenotype of individuals with 3q26-3q28 microdeletions is heterogeneous: IUGR, postnatal growth impairment, feeding problems, short stature, dysmorphic facial features, microcephaly, seizure, dental and limb abnormalities, developmental delay, intellectual disability, hypotonia, and thrombocytopenia. Additionally, in the Decipher database, several individuals harboring the deletions (range of $1.8-2.7 \mathrm{Mb}$ ) in this region has been reported to exhibit variety of phenotypes including intrauterine growth retardation, short stature, microcephaly, facial dysmorphisms, hypotonia, developmental delay, and cardiac defects (DECIPHER ID: 317983, 276986, and 323724). While there is some degree of phenotypic variability that primarily relates to the size of the deletion, the most striking clinical features shared among all reported cases are prenatal and postnatal growth restriction, as well as neurodevelopmental abnormalities. The clinical presentation of two patients described in this study supports the clinical profile described for other individuals in the literature (Table 1). The genotype-phenotype correlations for loss of the 3q26q28 region are, however, restricted by the fact that these individuals do not share common break points, like those generated in recurring pathogenic CNVs flanked by segmental duplications. Nonetheless, comparison of the clinical and molecular findings in Proband-1 and Proband-2 with the previous reported individuals suggests that this is a microdeletion syndrome with shared clinical features. Though the precise size and position of these deletions are uncertain, it has been proposed that haploinsufficiency of dosage sensitive genes leads to defined clinical sequelae [7].

By comparing microarray findings of these ten cases, we mapped the SRO to a size of $1.2 \mathrm{Mb}$, corresponding to genomic coordinates 183,220,510-184,469,308 (hg19) at chromosomal band 3q27.1 (Fig. 1). Deletions overlapping with this region are absent from population databases including gnomAD SVs v2.1 (controls) and DGV Gold Standard [14, 15]. This SRO region contains 46 genes, 24 of which are OMIM-annotated and eight of which are associated with disease (KLHL24, EIF2B5, DVL3, AP2M1, ALG3, EIF4G1, CLCN2, and THPO) (Additional file 2 Table S1). Among these genes, DVL3 is the most interesting one. Heterozygous pathogenic variants in the DVL3 gene have been associated with autosomal dominant type III Robinow syndrome (MIM\#: 616894), which shares many clinical features with the 3q26q28 microdeletion syndrome: short stature (9/9), facial dysmorphic features (10/10), teeth abnormalities (7/8), and hand abnormalities (6/10). Emerging data suggest DVL3 is a core component in the routing and transmission of canonical and non-canonical Wnt signalasome [16]. In murine, DVL3 has been detected to express ubiquitously at E7.5, but shortly after it showed elevated expression in heart, CNS, notochord, dorsal root ganglia, branchial arches, limb buds, and somitic mesoderm $[17,18]$. These findings further strengthen the role of DVL3 in diseases by modulating Wnt signaling that is involve in cell migration and tissue morphogenesis in vertebrate development. Indeed, $D v l 3$ knock out mice demonstrated partial lethality, conotruncal defects and neural tube defects, 


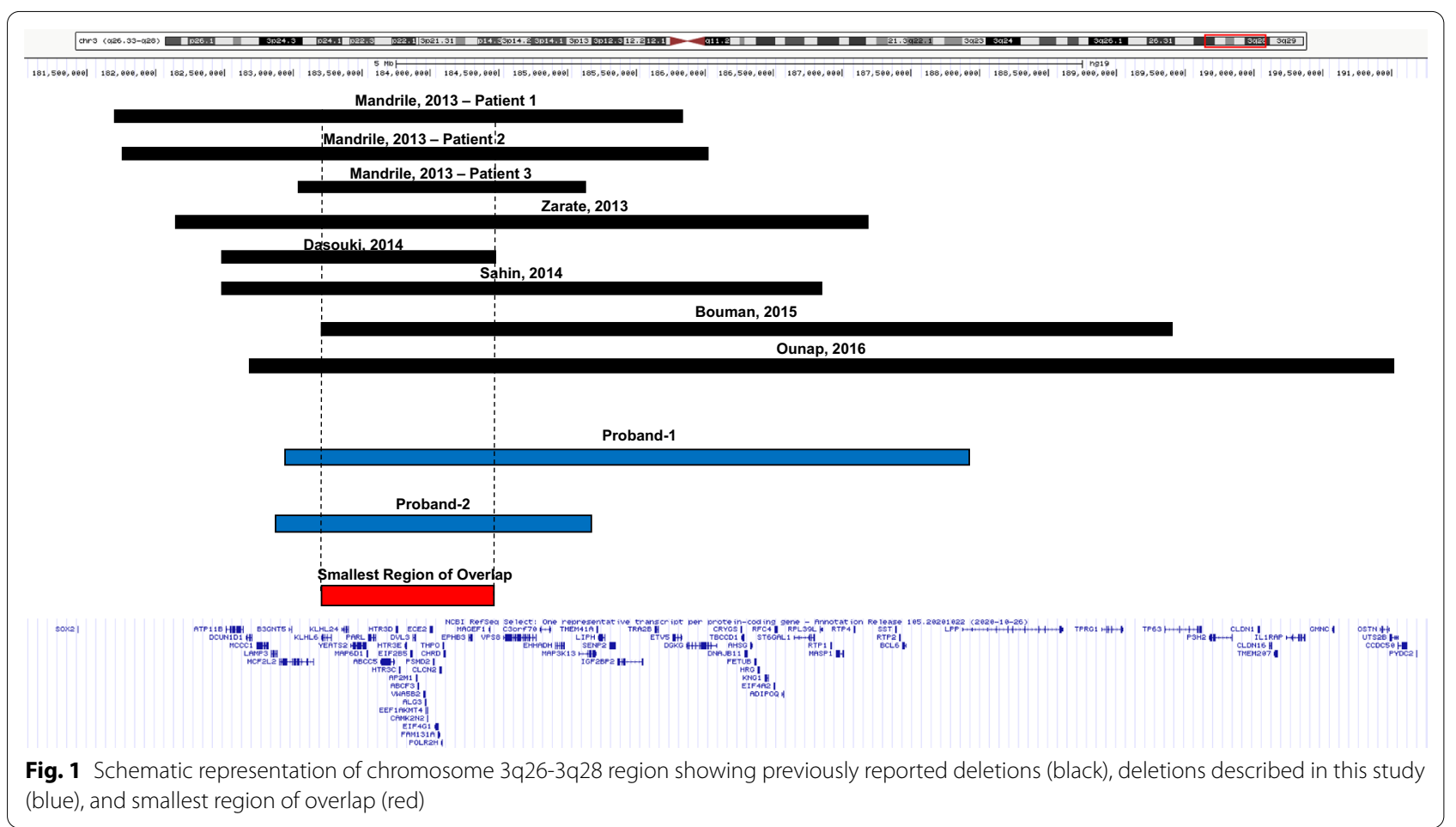

including abnormalities in cochlear cells [19]. However, all current known pathogenic variants of $D V L 3$ are frameshift small insertions/deletions or splice variants in the last two exons; and larger intergenic deletions of DVL3 have not been described previously [20-22]. Furthermore, it was demonstrated by expression studies that truncating $D V L 3$ variants escape nonsense-mediated decay (NMD), suggesting a dominant-negative or gain-of-function disease mechanism [21-24]. Therefore, the exact contribution from loss of $D V L 3$ to phenotype caused by $3 q 26-3 q 28$ microdeletions is still uncertain at this time.

Beside DVL3, the role of AP2M1 (MIM\#: 601024) in developmental delay (9/9), hypotonia (6/9), and seizures (2/9), as well as the role of PARL (MIM\#: 607858) in growth restriction $(10 / 10)$ are of great interest. AP2M1 has recently been associated with impaired intellectual development, poor speech, and delayed walking [25]. Though a recurrent missense variant in $A P 2 M 1$ has been reported, $A P 2 M 1$ is highly intolerant to loss-of-function variant in general population with a probability of intolerance to loss of function (pLI) of 1.0 and the Haploinsufficiency Score of 8.13. Previous studies with Parl knock out mouse model have shown that Parl plays an essential physiological role in the neurological homeostasis [26], and Parl deficiency results in growth retardation, cachexia, and severe atrophy of skeletal muscle, thymus, and spleen [27]. However, we think the growth phenotype caused by this SRO is predominantly overlap with $D V L 3$ related Robinow syndrome and further study is warranted to associate the role of PARL in this phenotype. The remaining OMIM genes in the SRO ( $A L G 3$, CLCN2, EIF2B5, EIF4G1, KLHL24, and THPO) are associated with autosomal recessive conditions and therefore are less likely to have major contributions to these patients' phenotype.

In order to evaluate the clinical significance of the SRO, we further assessed the deduced SRO corresponding to genomic coordinates, chr3:183,220,510-184,469,308 (hg19) using ACMG/ ClinGen current recommendations for classifying copy number variants (CNVs) [13]. This SRO harbors 26 protein coding RefSeq genes (Criteria $3 \mathrm{~B}$, points given: 0.45 ). Three of them, PSMD2, AP2M1, and EIF4G1 are predicted to be haploinsufficiency genes by the gnomAD pLI score [28], the gomAD LOEUF score [29], and the DECIPHER HI index [30] (Additional file 2: Table S1; Criteria $2 \mathrm{H}$, points given: 0.15 ).. To the best of our knowledge, in the literature the SRO overlap with four previously assumed (due lack of molecular confirmation for paternity and maternity) de novo cases (individuals $1,2,5$, and 7 in Table 1) with phenotype that is consistent with the gene/genomic region, but not highly 
specific and/or with high genetic heterogeneity (Criteria $4 \mathrm{C}$, points given: 0.40 ). Moreover, observed copy number loss is assumed de novo (due lack of molecular confirmation for paternity and maternity) for Proband-1 in this study (Criteria 5A, points given: 0.1). Using these recommendations as a framework, we classified the SRO as pathogenic (Total score: 1.1).

In conclusion, in this study we present two additional individuals with phenotype similar to previously reported cases with overlapped deletions in chromosome 3q26q28 region. It provides further evidence supporting the existence of this novel microdeletion syndrome. Additionally, our molecular cytogenetic and clinical findings defined the 1.2 Mb SRO at chromosomal band 3q27.1 as the critical region for this microdeletion syndrome. The refinement of this critical region suggests that deletion of at least three genes (DVL3, PARL and AP2M1) may contribute to anomalies observed in these individuals. At last, we utilized the new ACMG/Clingen standards for $\mathrm{CNV}$ interpretation with refined molecular mapping that improved our ability for clinical diagnosis and genetic counselling of individuals harboring similar imbalance.

\section{Abbreviations}

CNV: Copy number variants; IUGR: Intrauterine growth restriction; NMD: Nonsense-mediated decay; OFC: Occipitofrontal circumference; SNP: Single nucleotide polymorphism; SOMA: Single nucleotide polymorphism oligonucleotide microarray analysis; SRO: Smallest region of overlap.

\section{Supplementary Information}

The online version contains supplementary material available at https://doi. org/10.1186/s13039-022-00587-0.

Additional file 1: Figure S1. Test results for Proband-1 and his parents. a SOMA result showing the $3 \mathrm{q} 27.1 \mathrm{q} 28$ deletion in the Proband-1. b Normal SOMA result from the mother. $\mathbf{c}$ FISH result using the BAC probe RP11919L13 confirmed the presence of the deletion in Proband-1. d Normal FISH result from the father. Figure S2. Test results for Proband- 2 and her mother. a SOMA result showing the 3q27.1q27.2 deletion in Proband-2. b FISH result using the BAC probe RP11-919L13 confirmed the presence of the deletion in Proband-2. c Normal FISH result from the mother (PPTX $714 \mathrm{~KB})$

Additional file 2: Table S1. Summary of protein coding genes within the $1.2 \mathrm{Mb}$ in chromosome 3q27.1 (XLSX $13 \mathrm{~KB}$ )

\section{Acknowledgements}

We would like to thank patients and their families for their participation.

\section{Authors' contributions}

SB wrote the first draft of the manuscript. EMP and K A-Y obtained clinical information. SB, VJ, BL, and JL performed genetic analysis and data interpretation. JL reviewed and critically revised the manuscript before submission. All authors read and approved the final manuscript.

\section{Funding}

None.

\section{Availability of data and materials}

The datasets used and/or analysed during the current study are available from the corresponding author on reasonable request.

\section{Declarations}

Ethical approval and consent for participate

This study was approved by Institutional Review Board of Columbia University Medical Center. All information in this report has been de-identified in accordance with HIPAA and institutional review board regulations.

\section{Consent for publication}

Informed consent was obtained from parents of patients in this study.

\section{Competing interests}

The authors declare that they have no competing interests.

\section{Author details}

${ }^{1}$ Department of Pathology, Anatomy, and Laboratory Medicine, West Virginia University Health Sciences Center, Morgantown, WV, USA. ${ }^{2}$ Division of Clinical Genetics, Department of Pediatrics, Columbia University Medical Center, New York, NY, USA. ${ }^{3}$ Department of Pathology and Cell Biology, Columbia University Medical Center, New York, NY, USA.

Received: 27 August 2021 Accepted: 9 February 2022

Published online: 03 March 2022

\section{References}

1. Roifman M, Choufani S, Turinsky AL, Drewlo S, Keating S, Brudno M, et al. Genome-wide placental DNA methylation analysis of severely growthdiscordant monochorionic twins reveals novel epigenetic targets for intrauterine growth restriction. Clin Epigenetics. 2016;8:70.

2. Sagi-Dain L, Peleg A, Sagi S. Risk for chromosomal aberrations in apparently isolated intrauterine growth restriction: a systematic review. Prenat Diagn. 2017;37(11):1061-6.

3. Sharma D, Sharma P, Shastri S. Genetic, metabolic and endocrine aspect of intrauterine growth restriction: an update. J Matern Fetal Neonatal Med. 2017;30(19):2263-75.

4. Beaumann M, Delhaes F, Menetrey S, Joye S, Vial Y, Baud D, et al. Intrauterine growth restriction is associated with sex-specific alterations in the nitric oxide/cyclic GMP relaxing pathway in the human umbilical vein. Placenta. 2020;93:83-93.

5. Borrell A, Grande M, Meler E, Sabria J, Mazarico E, Munoz A, et al. Genomic microarray in fetuses with early growth restriction: a multicenter study. Fetal Diagn Ther. 2017;42(3):174-80.

6. Borrell A, Grande M, Pauta M, Rodriguez-Revenga L, Figueras F. Chromosomal microarray analysis in fetuses with growth restriction and normal karyotype: a systematic review and meta-analysis. Fetal Diagn Ther. 2018;44(1):1-9.

7. Mandrile G, Dubois A, Hoffman JD, Uliana V, Di Maria E, Malacarne M, et al 3q26.33-3q27.2 microdeletion: a new microdeletion syndrome? Eur J Med Genet. 2013;56(4):216-21.

8. Zarate YA, Bell C, Schaefer B. Description of another case of 3q26.333q27.2 microdeletion supports a recognizable phenotype. Eur J Med Genet. 2013;56(11):624-5.

9. Dasouki M, Roberts J, Santiago A, Saadi I, Hovanes K. Confirmation and further delineation of the 3q26.33-3q27.2 microdeletion syndrome. Eur J Med Genet. 2014;57(2-3):76-80.

10. Sahin Y, Kiper PO, Alanay Y, Liehr T, Utine GE, Boduroglu K. Partial monosomy 3q26.33-3q27.3 presenting with intellectual disability, facial dysmorphism, and diaphragm eventration: a case report. Clin Dysmorphol. 2014;23(4):147-51.

11. Bouman A, Weiss M, Jansen S, Hankel M, Nieuwint A, Adriaanse B, et al. An interstitial de-novo microdeletion of 3q26.33q27.3 causing severe intrauterine growth retardation. Clin Dysmorphol. 2015;24(2):68-74. 
12. Ounap K, Pajusalu S, Zilina O, Reimand T, Zordania R. An 8.4-Mb 3q26.33$3 \mathrm{q} 28$ microdeletion in a patient with blepharophimosis-intellectual disability syndrome and a review of the literature. Clin Case Rep. 2016;4(8):824-30.

13. Riggs ER, Andersen EF, Cherry AM, Kantarci S, Kearney H, Patel A, et al. Technical standards for the interpretation and reporting of constitutional copy-number variants: a joint consensus recommendation of the American College of Medical Genetics and Genomics (ACMG) and the Clinical Genome Resource (ClinGen). Genet Med. 2020:22(2):245-57.

14. Collins RL, Brand H, Karczewski KJ, Zhao X, Alfoldi J, Francioli LC, et al. A structural variation reference for medical and population genetics. Nature. 2020;581(7809):444-51.

15. MacDonald JR, Ziman R, Yuen RK, Feuk L, Scherer SW. The database of genomic variants: a curated collection of structural variation in the human genome. Nucleic Acids Res. 2014;42(Database issue):D986-92.

16. Gentzel M, Schambony A. Dishevelled paralogs in vertebrate development: redundant or distinct? Front Cell Dev Biol. 2017;5:59.

17. Tsang M, Lijam N, Yang Y, Beier DR, Wynshaw-Boris A, Sussman DJ. Isolation and characterization of mouse dishevelled-3. Dev Dyn. 1996;207(3):253-62

18. Diez-Roux G, Banfi S, Sultan M, Geffers L, Anand S, Rozado D, et al. A highresolution anatomical atlas of the transcriptome in the mouse embryo. PLoS Biol. 2011;9(1): e1000582.

19. Etheridge SL, Ray S, Li S, Hamblet NS, Lijam N, Tsang M, et al. Murine dishevelled 3 functions in redundant pathways with dishevelled 1 and 2 in normal cardiac outflow tract, cochlea, and neural tube development. PLoS Genet. 2008;4(11): e1000259.

20. White JJ, Mazzeu JF, Hoischen A, Bayram Y, Withers M, Gezdirici A, et al. DVL3 alleles resulting in a -1 frameshift of the last exon mediate autosomal-dominant robinow syndrome. Am J Hum Genet. 2016;98(3):553-61.

21. Danyel M, Kortum F, Dathe K, Kutsche K, Horn D. Autosomal dominant Robinow syndrome associated with a novel DVL3 splice mutation. Am J Med Genet A. 2018;176(4):992-6.

22. White JJ, Mazzeu JF, Coban-Akdemir Z, Bayram Y, Bahrambeigi V, Hoischen $A$, et al. WNT signaling perturbations underlie the genetic heterogeneity of Robinow syndrome. Am J Hum Genet. 2018;102(1):27-43.

23. White J, Mazzeu JF, Hoischen A, Jhangiani SN, Gambin T, Alcino MC, et al. DVL1 frameshift mutations clustering in the penultimate exon cause autosomal-dominant Robinow syndrome. Am J Hum Genet. 2015;96(4):612-22.

24. Bunn KJ, Daniel P, Rosken HS, O'Neill AC, Cameron-Christie SR, Morgan T, et al. Mutations in DVL1 cause an osteosclerotic form of Robinow syndrome. Am J Hum Genet. 2015;96(4):623-30.

25. Helbig I, Lopez-Hernandez T, Shor O, Galer P, Ganesan S, Pendziwiat M, et al. A recurrent missense variant in AP2M1 impairs clathrin-mediated endocytosis and causes developmental and epileptic encephalopathy. Am J Hum Genet. 2019;104(6):1060-72.

26. Spinazzi M, Radaelli E, Horre K, Arranz AM, Gounko NV, Agostinis $P$, et al. PARL deficiency in mouse causes Complex III defects, coenzyme Q depletion, and Leigh-like syndrome. Proc Natl Acad Sci U S A. 2019;116(1):277-86

27. Cipolat S, Rudka T, Hartmann D, Costa V, Serneels L, Craessaerts K, et al. Mitochondrial rhomboid PARL regulates cytochrome $c$ release during apoptosis via OPA1-dependent cristae remodeling. Cell. 2006;126(1):163-75

28. Lek M, Karczewski KJ, Minikel EV, Samocha KE, Banks E, Fennell T, et al. Analysis of protein-coding genetic variation in 60,706 humans. Nature. 2016;536(7616):285-91.

29. Karczewski KJ, Francioli LC, Tiao G, Cummings BB, Alfoldi J, Wang Q, et al. The mutational constraint spectrum quantified from variation in 141,456 humans. Nature. 2020;581(7809):434-43.

30. Huang N, Lee I, Marcotte EM, Hurles ME. Characterising and predicting haploinsufficiency in the human genome. PLoS Genet. 2010;6(10): e1001154.

\section{Publisher's Note}

Springer Nature remains neutral with regard to jurisdictional claims in published maps and institutional affiliations.

Ready to submit your research? Choose BMC and benefit from:

- fast, convenient online submission

- thorough peer review by experienced researchers in your field

- rapid publication on acceptance

- support for research data, including large and complex data types

- gold Open Access which fosters wider collaboration and increased citations

- maximum visibility for your research: over $100 \mathrm{M}$ website views per year

At BMC, research is always in progress.

Learn more biomedcentral.com/submissions 